\title{
Surgical management of chronic pelvic pain
}

\author{
Kamal Ojha' ${ }^{2}$ Arti Matah ${ }^{1}$ \\ Sri Lanka Journal of Obstetrics and Gynaecology 2009; 31: 59-65
}

This article is reproduced with the kind permission of the authors and the publisher, Elsevier.

\begin{abstract}
Chronic pelvic pain is a common debilitating condition affecting women and presents a major challenge to healthcare providers. Laparoscopy is the only test capable of diagnosing peritoneal endometriosis and adhesions. Gynaecologists have, therefore, seen it as an essential tool in the assessment of women with chronic pelvic pain. This article explores the role of surgery in the management of chronic pelvic pain. The approach to women with chronic pain must be therapeutic, supportive and sympathetic. Depending on the severity of disease found, ideal practice is to diagnose and remove the disease surgically at the same time during laparoscopy. A maximum surgical effort must be expended to resect all endometriosis, restore normal pelvic anatomy, resect nerve fibres and treat surgically accessible disease.
\end{abstract}

Keywords: chronic pelvic pain (CPP); endometriosis; laparoscopy; pelvic adhesions

\section{Introduction}

Chronic pelvic pain (CPP) is a source of frustration to both the physician and the patient. It is a common debilitating condition affecting women and presents a major challenge to healthcare providers because of its unclear aetiology, complex natural history and poor response to therapy. Living with any chronic pain carries a heavy economic and social price.

CPP can be defined as intermittent or constant pain in the lower abdomen or pelvis of at least 6 months' duration, not occurring exclusively with menstruation or intercourse and not associated with pregnancy. It is a symptom, not a diagnosis. CPP presents in primary care as frequently as migraine or lower back pain.

\footnotetext{
${ }^{1}$ Consultant in Obstetrics and Gynaecology, St George's Hospital and Medical School, London, UK

${ }^{2}$ Clinical Fellow, Department of Obstetrics and Gynaecology, St George's Hospital and Medical School, London, UK.
}

CPP is common, affecting perhaps one in six of the adult female population. It is the reason for $10 \%$ of all office visits to a gynaecologist and for over $40 \%$ of laparoscopies performed by gynaecologists.

The pelvis can be compared with a grand central station. There is frequently more than one component to CPP. The approach to the patient with CPP must take into account the major sources of the origin of this pain:

- gynaecological

- psychological

- musculoskeletal

- urological

- gastrointestinal

- neurological.

Only by addressing and evaluating each of these components by a very careful history and physical examination and by approaching the patient in a comprehensive manner can the source of the pain be determined and appropriate therapy provided. The differential diagnosis of CPP is outlined in Table 1.

CPP is poorly understood and, consequently, poorly managed. There is increasing awareness that CPP cannot be successfully managed using a simplistic approach. This condition is best managed using a multidisciplinary approach. Management requires good integration and knowledge of all pelvic organ systems and other systems including musculoskeletal, neurologic and psychiatric systems. There are important questions about the optimal use of this approach as it is time-consuming and expensive. Realistically, many cases will continue to be seen by a single specialist, emphasising the need for the skills relevant to CPP being embedded in gynaecological specialist training.

Adequate time should be allowed for the initial assessment of women with CPP. They need to feel that they have been able to tell their story and that they have been listened to and believed. This may be therapeutic in itself. The multifactorial nature of CPP should be discussed and explored from the start. The aim should be to develop a partnership between clinician and patient to plan a management programme. 
Patient history is important in cases of CPP. Because of the complex aetiology and, often, the presence of associated disorders, a general and clinically oriented approach with a thorough history and systematic examination that directs further evaluation and appropriate consultations is needed. The initial history should include questions about the pattern of the pain and its association with other problems, such as psychological, bladder and bowel symptoms, and the effect of movement and posture on the pain. A thorough past history is also important to avoid repeating invasive and expensive procedures. Completing a daily pain diary for two to three menstrual cycles may help the woman and the doctor identify provoking factors or temporal associations. The information may be useful in understanding the cause of the pain. Suitable samples to screen for infection, particularly Chlamydia and gonorrhoea, should be taken if there is any suspicion of pelvic inflammatory disease. Ideally, all sexually active women below the age of 25 years who are being examined should be offered opportunistic screening for Chlamydia.

Many women with CPP can be managed in primary care. General practitioners might consider referral when the pain has not been explained to the woman's satisfaction or when pain is inadequately controlled. If the history suggests to patient and doctor that there is a non-gynaecological component to the pain, referral to the relevant healthcare professional such as gastroenterologist, urologist, genitourinary medicine physician, physiotherapist, psychologist or psychosexual counsellor should be considered.

Table 1. Causes of chronic pelvic pain

\begin{tabular}{|c|c|c|c|c|c|}
\hline Gynaecologic & Gastrointestinal & Genitourinary & Neurologic & Musculoskeletal & Systemic \\
\hline $\begin{array}{l}\text { Non-cyclic } \\
\text { - Adhesions } \\
\text { - Endometriosis } \\
\text { - Salpingo-oophoritis } \\
\text { - Ovarian remnant or } \\
\text { retained ovary syndrome } \\
\text { - Pelvic congestion } \\
\text { - Ovarian neoplasm benign } \\
\text { or malignant } \\
\text { - Pelvic relaxation } \\
\text { Cyclic } \\
\text { - Primary dysmenorrhoea } \\
\text { - Mittelschmerz } \\
\text { - Secondary dysmenorrhoea } \\
\text { - Undometriosis } \\
\text { with obstruction of menstrual } \\
\text { outflow } \\
\text { - Intrauterine synechiae } \\
\text { (Asherman's syndrome) } \\
\text { - Endometrial polyps or } \\
\text { intrauterine device } \\
\text { - Uterine leiomyomata } \\
\text { - Adenomyosis } \\
\text { - Pelvic congestion syndrome } \\
\text { formation }\end{array}$ & $\begin{array}{l}\text { - Irritable bowel } \\
\text { syndrome } \\
\text { - Ulcerative colitis } \\
\text { - Granulomatous } \\
\text { colitis (Crohn's } \\
\text { disease) } \\
\text { - Carcinoma } \\
\text { - Infection } \\
\text { - Recurrent } \\
\text { partial bowel } \\
\text { obstruction } \\
\text { - Diverticulitis } \\
\text { - Hernia } \\
\text { - Recurrent } \\
\text { appendiceal colic }\end{array}$ & $\begin{array}{l}\text { - Recurrent } \\
\text { or relapsing } \\
\text { cystourethritis } \\
\text { - Urethral } \\
\text { syndrome } \\
\text { - Interstitial } \\
\text { cystitis } \\
\text { - Urethral } \\
\text { diverticulitis or } \\
\text { polyps } \\
\text { - Carcinoma of } \\
\text { the bladder } \\
\text { - Ureteral } \\
\text { obstruction } \\
\text { - Pelvic kidney }\end{array}$ & $\begin{array}{l}\text { - Nerve } \\
\text { entrapment } \\
\text { syndrome, } \\
\text { neuroma, } \\
\text { or other } \\
\text { neuropathies } \\
\text { - Trigger points }\end{array}$ & $\begin{array}{l}\text { Low back pain } \\
\text { syndrome } \\
\text { - Congenital } \\
\text { anomalies } \\
\text { - Scoliosis and } \\
\text { kyphosis } \\
\text { - Spondylolysis } \\
\text { - Spondylolisthesis } \\
\text { - Spinal injuries } \\
\text { - Inflammation } \\
\text { - Tumours } \\
\text { - Osteoporosis } \\
\text { - Degenerative } \\
\text { changes } \\
\text { - Coccydynia } \\
\text { - Myofascial } \\
\text { syndrome }\end{array}$ & $\begin{array}{l}\text { - Fibromyalgia } \\
\text { - Acute intermittent } \\
\text { porphyria } \\
\text { - Abdominal } \\
\text { migraine } \\
\text { - Connective tissue } \\
\text { disease including } \\
\text { systemic lupus } \\
\text { erythematosus } \\
\text { - Lymphoma } \\
\text { - Neurofibromatosis }\end{array}$ \\
\hline
\end{tabular}


Women should be offered appropriate analgesia to control their pain. Regular non-steroidal antiinflammatory drugs with or without paracetamol may be particularly useful in this context. Compound analgesics such as co-dydramol may be appropriate. Amitriptyline or gabapentin may be useful agents for the treatment of neuropathic pain. Non-pharmacological modalities such as transcutaneous nerve stimulation, acupuncture and other complementary therapies may also be helpful for some women. Dietary modification could also relieve pain. Women with cyclical pain should be offered a therapeutic trial using the combined oral contraceptive pill or a gonadotrophin-releasing hormone $(\mathrm{GnRH})$ agonist for a period of 3.6 months before having a diagnostic laparoscopy. The levonorgestrel-releasing intrauterine system could also be considered. Ovarian suppression can be an effective treatment for pain associated with endometriosis. Response to ovarian suppression can, therefore, be a useful diagnostic tool. The effect can be achieved with combined oral contraceptives, progestogens, danazol or GnRH agonists, all of which are equally effective but have different adverse effect profiles. Other proposed causes of cyclical pain, such as pelvic venous congestion, also appear to be wellcontrolled by ovarian suppression.

This article discusses in detail the surgical management of pelvic pain.

\section{Management}

Treatment of CPP must be tailored for the individual patient and the goals of treatment must be realistic. Focused towards restoration of normal function (minimal disability), better quality of life and prevention of relapse of chronic symptoms

\section{Investigations}

\section{Laparoscopy}

Diagnostic laparoscopy has been regarded in the past as the gold standard in the diagnosis of CPP. Laparoscopy is the only test capable of diagnosing peritoneal endometriosis and adhesions. Gynaecologists have, therefore, seen it as an essential tool in the assessment of women with CPP. However, it carries significant risks: an estimated risk of death of approximately $1.0 / 10,000$ and a risk of injury to bowel, bladder or blood vessel of approximately 2.4/ 1000 , of whom two-thirds will require laparotomy. Clearly, conditions such as irritable bowel syndrome and adenomyosis are not visible at laparoscopy but there is also a risk that some forms of endometriosis will be missed.
Good surgical practice is to use an instrument such as a grasper, via a secondary port, to mobilise the pelvic organs and to palpate lesions, which can help determine their nodularity. It is also important to document in detail the type, location and extent of all lesions and adhesions in the operative notes. Ideal practice is to record the findings on video or DVD. All suspicious areas should be biopsied. Positive histology confirms the diagnosis of endometriosis; negative histology does not exclude it. Visual inspection is usually adequate but histological confirmation of at least one lesion is ideal. In cases of ovarian endometrioma (greater than $3 \mathrm{~cm}$ in diameter) and in deeply infiltrating disease, histology should be obtained to identify endometriosis and to exclude rare instances of malignancy.

The findings of one-third to one-half of diagnostic laparoscopies will be negative and much of the pathology identified is not necessarily the cause of pain. The risks and benefits of diagnostic laparoscopy and the possibility of negative findings should be discussed before the decision is made to perform a laparoscopy. Perhaps it should only be performed when the index of suspicion of adhesive disease or endometriosis requiring surgical intervention is high, or when the woman has specific concerns that could be addressed by diagnostic laparoscopy, such as the existence of endometriosis or adhesions potentially affecting her fertility.

Microlaparoscopy or conscious pain mapping has been proposed as an alternative to diagnostic laparoscopy under general anaesthesia. Although the technique seems to provide an opportunity to confirm particular lesions as the source of the woman $\bullet$ fs pain, it has not been widely adopted in the UK and questions remain as to the acceptability, reproducibility and validity of this technique.

\section{Imaging}

Transvaginal scanning is an appropriate investigation to screen for and assess adnexal masses. A systematic review of the value of transvaginal scanning in the diagnosis of endometriosis found that endometriomas may be accurately distinguished from other adnexal masses with a positive likelihood ratio of 8-30. Transvaginal scanning is of little value for the assessment of other causes of CPP, including peritoneal endometriosis. Transvaginal scanning and magnetic resonance imaging are useful tests to diagnose adenomyosis.

\section{Surgical management}

Surgical approaches to the management of CPP can be termed radical or conservative. Most 
conservative surgery is currently accomplished via the laparoscopic approach.

\section{Laparoscopy}

Typical laparoscopic findings in women investigated for CPP are in increasing order of frequency . adhesions (24\%), endometriosis (33\%) and 'no pathology, (35\%).

\section{Endometriosis-associated pain}

Objectives of treatment are to restore normal anatomy, remove or ablate endometrial implants and prevent or delay recurrence of disease. Surgery involves the excision/ablation of endometriotic lesions plus adhesiolysis. Improvements in equipment and operative technique have made laparoscopy the preferred surgical approach. There is less tissue trauma, improved visualisation, less adhesion formation and early recovery.

\section{Localisation and appearance of endometriosis}

Endometriosis is a disease with a large variety of appearances and many authorities consider that it is significantly under-diagnosed at laparoscopy. It is well known that the existing scoring systems do not correlate with severity of pain and that deeply infiltrating endometriosis, which is strongly correlated with pain, may be misinterpreted as minimal disease.

The most commonly affected sites are the pelvic organs and peritoneum, although other parts of the body such as the bowel or lungs are occasionally affected. The extent of the disease varies from a few, small lesions on otherwise normal pelvic organs to large, ovarian endometriotic cysts (endometriomas). There can be extensive fibrosis in structures - such as the uterosacral ligaments- and adhesion formation causing marked distortion of pelvic anatomy.

Endometriosis typically appears as superficial 'powder-burn' or 'gunshot' lesions on the ovaries, serosal surfaces and peritoneum: black, dark-brown or bluish puckered lesions, nodules or small cysts containing old haemorrhage surrounded by a variable extent of fibrosis. Atypical or 'subtle' lesions are also common, including red implants (petechial, vesicular, polypoid, hemorrhagic, red flame-like) and serous or clear vesicles. Other appearances include white plaques or scarring and yellow-brown peritoneal discoloration of the peritoneum.

Endometriomas usually contain thick fluid, like chocolate. Such cysts are often densely adherent to the peritoneum of the ovarian fossa and the surrounding fibrosis may involve the tubes and bowel. Deeply infiltrating endometriotic nodules extend more than $5 \mathrm{~mm}$ beneath the peritoneum and may involve the uterosacral ligaments, vagina, bowel, bladder or ureters. The depth of infiltration is related to the type and severity of symptoms.

Depending on the severity of disease found, ideal practice is to diagnose and remove endometriosis surgically at the same time as investigation, provided that pre-operative adequate consent has been obtained. There is no data to justify hormonal treatment prior to surgery to improve the success of surgery.

\section{Peritoneal endometriosis}

Endometriotic lesions can be removed during laparoscopy by surgical excision with scissors, bipolar coagulation or laser methods $\left(\mathrm{CO}_{2}\right.$ laser, argon laser etc.).

The results that can be anticipated with various laparoscopic techniques are confounded by a number of factors. Firstly, visualisation of endometriosis lesions at the time of laparoscopy does not correlate with histological confirmation of endometriosis. Secondly, there is a complete absence of correlation between findings at laparoscopy and the severity of symptoms. Thirdly, differences in surgical technique (ablation compared with excision) have not been studied in any well-designed, prospective, comparative fashion.

A review of the role of laparoscopic surgery in the treatment of endometriosis concluded that laser laparoscopic cytoreduction of ectopic endometrial implants offers a reasonable degree of pain relief in mild, minimal and moderate disease. A total of $12 \%$ of patients who suffered from recurrent disease required repeat laparoscopic surgery. The recurrences arose de novo and rarely occurred at previously treated sites unless the surgeon failed to remove deeply infiltrating disease completely in the uterosacral ligaments or the rectovaginal septum. Complete surgical eradication of the disease resulted in pain relief in $81 \%$ of patients whose pain was due to endometriosis. However, $19 \%$ experienced recurrence of new disease in 5 years.

\section{Ovarian endometriosis}

Operative laparoscopic management is the 'gold standard' for surgical treatment. The size of the ovarian cyst is most important. Superficial ovarian lesions can be vaporised. Small ovarian endometrioma ( $<3 \mathrm{~cm}$ in diameter) can be aspirated and irrigated; their interior wall can be vaporised to destroy the 
mucosal lining of the cyst. For cysts $<5 \mathrm{~cm}$, the cyst wall can generally be stripped easily from the ovary. The cyst should be aspirated followed by incision and removal of the cyst wall from the ovarian cortex. The ovarian capsule around the cyst opening is incised with $\mathrm{CO}_{2}$ laser. Once the plane of cleavage is found, the cyst wall is easily stripped from the ovary. To prevent recurrence, the cyst wall of the endometrioma must be removed and normal ovarian tissue must be preserved. Based on current evidence, ovarian cystectomy appears to be the method of choice. Some concern has been reported that ovarian cystectomy may result in inadvertent removal or destruction of primordial follicles at the same time and thus reduce ovarian volume and reserve, and diminish fertility. To avoid this, replacing cystectomy by fenestration and coagulation of the inner wall of the endometriotic ovarian cyst has been suggested.

For larger cysts, the pragmatism of size practically excludes excision and/or vaporisation. A two-stage procedure can be performed. At the first operation, a large window is made in the cyst wall, followed by rinsing, some focal treatment is given, adhesiolysis, and then 3 months of $\mathrm{GnRH}$ agonist treatment is given postoperatively. If on ultrasound the cyst persists or reforms, this small cyst is then treated using a second operation by excision. If no cyst is found, a second intervention may not be necessary in the absence of pain or infertility.

The outcome of surgical therapy in patients with endometriosis and pain is influenced by many psychological factors relating to personality, depression and marital and sexual problems. Although there are limited data available from randomised controlled trials assessing the effectiveness of surgery in relieving pain, it is clearly effective for many women. There is also a significant placebo response to surgical therapy; diagnostic laparoscopy with complete removal of endometriosis may alleviate pain in $50 \%$ of patients. However, clinical experience shows that some women fail to respond to surgical treatment either because of incomplete excision, postoperative disease recurrence or because some of their pain was not due to endometriosis in the first place.

Postoperative treatment with danazol or a GnRH agonist for 6 months after surgery reduces endometriosis-associated pain and delays recurrence at 12 months and 24 months compared with placebo and expectant management. However, postoperative treatment with a combined oral contraceptive is not effective.

\section{Pelvic adhesions}

A meta-analysis of over 3000 women with CPP and over 2000 controls showed that adhesions were present in $36 \%$ of women with CPP compared with $15 \%$ controls. However, the role of adhesions in CPP has been questioned. A retrospective study comparing asymptomatic infertile patients with women who had CPP did not reveal a significant difference in the density or location of adhesions. Some declare that it is an 'unsubstantiated myth' that adhesions cause pain while others think that adhesions can cause pelvic pain and adhesiolysis relieves it in $60.90 \%$ of cases. It, therefore, appears that some adhesions are associated with pain and some are not. It has been proposed that adhesions that are dense and vascular are more likely to result in pelvic pain.

A number of non-randomised studies have shown that division of adhesions at surgery is useful in the treatment of CPP. In terms of retrospective studies, a meta-analysis showed that out of over 600 patients with $\mathrm{CPP}, 76 \%$ would obtain relief from adhesiolysis. Adhesiolysis may be accomplished by blunt or sharp dissection with scissors, laser or an electrosurgical electrode. Another study reported that 55 of 65 patients $(84 \%)$ with chronic lower abdominal pain who underwent laser laparoscopic adhesiolysis experienced symptomatic relief.

A Cochrane review concluded that 'there is still uncertainty about the place of adhesiolysis among patients presenting to gynaecologists and the conclusion of this review is that there is no evidence of benefit, rather than evidence of no benefit'.

\section{Advanced laparoscopic surgery and laparotomy}

Laparotomy should be reserved for patients with advanced stage disease who cannot undergo a laparoscopic procedure and for those in whom fertility conservation is not necessary. It is more appropriate for the removal of a large endometrioma, extensive adhesiolysis or bowel resection. Deep nodular endometriosis may require partial rectal resection, which can be carried out laparoscopically.

The surgical excision of deep rectovaginal and rectosigmoidal endometriosis is difficult and can be associated with major complications. A preoperative ultrasound, contrast enema and intravenous pyelography are necessary in many cases together with a full preoperative bowel preparation. Therefore, if disease of such severity is suspected or diagnosed, referral to a centre with the necessary expertise to offer all available treatments in a multidisciplinary context including a gynaecologist, a colorectal surgeon and a urologist is strongly recommended. 
Surgery should be carefully planned. This planning comprises preoperative ureter stenting if gross ureteric distortion or hydronephrosis is present together with the eventual collaboration of a urologist to perform ureter re-anastomosis or repair, bladder suturing or ureter re-implantation. A colorectal surgeon is often required, since surgery can often extend from discoid excision with a muscularis defect, to resection of the rectum or sigmoid wall necessitating suture, to a large transmural nodule requiring a resection anastomosis if the defect is too large.

\section{Hysterectomy and oophorectomy}

Endometriosis-associated pain can be reduced by removing the entire lesions in severe and deeply infiltrating disease. If a hysterectomy is performed (can be performed either laparoscopically or more commonly by laparotomy) all visible endometriotic tissue should be removed at the same time. Bilateral salpingo-oophorectomy may result in improved pain relief and a reduced chance of future surgery. Hysterectomy is particularly useful for women who have completed childbearing and have secondary dysmenorrhoea or chronic pain related to endometriosis, to uterine pathology (such as adenomyosis) or to pelvic congestion. Hysterectomy for central pelvic pain in women with dysmenorrhoea, dyspareunia and uterine tenderness provided relief of pain in $77 \%$ of women in one retrospective study and $74 \%$ of women in one prospective cohort study. Nevertheless, $25 \%$ of women in the retrospective study noted a persistence or worsening of pain at 1 year follow-up.

Hormone replacement therapy (HRT) is recommended after bilateral oophorectomy in young women, given the overall health benefits and small risk of recurrent disease while taking HRT. The ideal regimen is unclear and should be discussed on an individual basis. Adding a progestogen after hysterectomy is unnecessary but may protect against the unopposed action of oestrogen on any residual disease. However, the theoretical benefit of avoiding disease reactivation or malignant transformation should be balanced against the increase in breast cancer risk reported to be associated with combined oestrogen and progestogen HRT and tibolone.

\section{Presacral neurectomy and laparoscopic uterine nerve ablation}

Presacral neurectomy (PSN) and laparoscopic uterine nerve ablation (LUNA) are both surgical procedures that involve the disruption of sensory nerve afferents that carry pain stimuli from the pelvis. In LUNA, the uterosacral ligaments are transacted close to their insertion at the cervix, thus interrupting part of the Lee- Franken-Hauser nerve plexus. In PSN, the presacral nerve plexus is isolated and cut proximally and distally. Complications associated with LUNA are rare . there have been isolated cases of uterine prolapse and bladder dysfunction. However, PSN has been associated with more serious complications . such as haematoma formation, major vessel injury as well as constipation and bladder dysfunction . although these complications are rare in experienced hands. Many uncontrolled studies have claimed LUNA and PSN to be effective for primary and secondary dysmenorrhoea. PSN may be indicated for primary and secondary dysmenorrhoea unrelieved by traditional therapy and unresponsive to multidisciplinary pain management. The response rate to PSN for secondary dysmenorrhoea varies between 50 and $75 \%$. In one study, LUNA was found to relieve dysmenorrhoea in $85 \%$ of patients. Shortterm results for PSN and LUNA for dysmenorrhoea seem to be similar, although PSN has better results in the long-term as suggested by the single trial comparing LUNA and PSN.

\section{Conclusions}

The approach to women with CPP must be therapeutic, supportive and sympathetic. An integrated approach that devotes attention to somatic, psychological, dietary, emotional and physiotherapeutic factors will likely show improvement over simply surgical or medical intervention.

A maximum surgical effort must be expended to resect all endometriosis, restore normal pelvic anatomy, resect nerve fibres and treat surgically accessible disease. A diagnostic/therapeutic trial of $\mathrm{GnRH}$ agonists is warranted prior to laparoscopic intervention in women with clinically diagnosed pelvic pain related to endometriosis. Rectovaginal, rectal, uterosacral lesions, endometrioma, abnormal anatomy and associated adhesions always require surgery.

CPP is a challenging problem. Thoroughness, continuity, multidisciplinary approach and compassion are central themes of successful management. The aim of the treatment must be pain reduction, improved quality of life and improved psychosocial functioning.

\section{Further reading}

Carter JE. Chronic pelvic pain diagnosis and management. OBGYN.net. Available at: www.obgyn.net/medical.asp?page = / english $/$ pubs/features/carter/cpp_carter 
Cheong Y, William Stones R. Chronic pelvic pain: aetiology and therapy. Best Pract Res Clin Obstet Gynaecol 2006; 20: 695.711.

Practice Committee of the American Society for Reproductive Medicine. Treatment of pelvic pain associated with endometriosis. Fertil Steril 2006; 86(Suppl. 5): S18.27.
Royal College of Obstetricians and Gynaecologists. The initial management of chronic pelvic pain. Guideline no. 41; April 2005. Available at: www.rcog.org.uk/index.asp?PageID = 1124

Royal College of Obstetricians and Gynaecologists. The investigation and management of endometriosis. Guideline no. 24; October 2006. Available at: www.rcog.org.uk/index.asp?PageID = 517

\section{Practice points}

- There is frequently more than one component to CPP.

Assessment should aim to identify contributory factors rather than assign causality to a single pathology

- Adequate time should be allowed for the initial assessment of women with CPP. They need to feel that they have been able to tell their story and that they have been listened to and believed

- The initial history should include questions about the pattern of the pain and its association with other problems, such as psychological, bladder and bowel symptoms, and the effect of movement and posture on the pain

- Ideal practice is to diagnose and treat endometriosis surgically

- Severe cases of endometriosis should be referred to units with the necessary expertise to offer all available treatments in a multidisciplinary context, including advanced laparoscopic surgery

- Hysterectomy with bilateral salpingooophorectomy is generally reserved for women with debilitating symptoms attributed to endometriosis who have completed childbearing and in whom other therapies have failed 